\title{
Order- $N$ electron transport calculations from ballistic to diffusive regimes by a time-dependent wave-packet diffusion method: Application to transport properties of carbon nanotubes
}

\author{
Hiroyuki Ishii* \\ National Institute of Advanced Industrial Science and Technology (AIST), Tsukuba Central 2, 1-1-1 Umezono, Tsukuba, \\ Ibaraki 305-8568, Japan \\ and Institute of Applied Physics, University of Tsukuba, 1-1-1 Tennodai, Tsukuba, Ibaraki 305-8573, Japan \\ Nobuhiko Kobayashi \\ Institute of Applied Physics, University of Tsukuba, 1-1-1 Tennodai, Tsukuba, Ibaraki 305-8573, Japan \\ Kenji Hirose \\ Nano Electronics Research Laboratories, NEC Corporation, 34 Miyukigaoka, Tsukuba, Ibaraki 305-8501, Japan \\ (Received 4 December 2009; revised manuscript received 17 June 2010; published 23 August 2010)
}

\begin{abstract}
We present an order- $N[O(N)]$ calculation method for the quantum electron transport of huge systems up to 80 million atoms. Based on the linear-response Kubo-Greenwood formula, we calculate the conductance through time-dependent diffusion coefficients using the time-dependent wave-packet diffusion approach, which treats the electron wave-packet motion with an $O(N)$ and very high-speed calculation. Combining with molecular-dynamics simulations, we can study the temperature dependence of electron transport properties of materials from atomistic viewpoints from ballistic to diffusive regimes. We apply the present calculation method to transport of the carbon nanotubes (CNTs) with various lengths at various temperatures. In metallic CNTs, the mean-free paths are in good agreements with recent experiments, which reach about $500 \mathrm{~nm}$ at room temperature and increase up to several micrometers at low temperature. We find that the resistance increases almost linearly with temperature and takes larger values than expected in the quasiballistic regime. In semiconducting CNTs, the mobilities are affected strongly by the contacts with metallic electrodes through Schottky barriers. The mobilities are maximally $30000 \mathrm{~cm}^{2} / \mathrm{V} \mathrm{s}$ and cut-off frequencies of $300 \mathrm{GHz}$ at room temperature. These calculated results provide useful information to the design of CNT field-effect-transistor devices.
\end{abstract}

DOI: 10.1103/PhysRevB.82.085435

PACS number(s): 72.10.-d, 72.15.Lh, 73.63.-b

\section{INTRODUCTION}

Recent advances in nanotechnology have made it possible to fabricate a number of new nanometer-scale devices using various materials such as atomic and molecular wires, ${ }^{1-3}$ silicon nanowires, ${ }^{4-6}$ carbon nanotubes $(\mathrm{CNTs}),{ }^{7-16}$ and graphenes. ${ }^{17-20}$ The transport properties change considerably depending on the sizes of these devices to compare with the mean-free path (MFP), above which the classical diffusive transport characters manifest, while below which the ballistic characters appear in such nanometer-scale systems. For the full understanding of the transport properties and to realize attractive functional devices with nanometer-scale lengths, it is important to study the electron transport from diffusive to ballistic regimes systematically.

In the diffusive transport regime, conductive electrons in the channel of electrical devices are scattered many times due to impurities and electron-phonon couplings, which result in the internal resistance proportional to the channel length. With decreasing the device size into the ballistic transport regime, such scattering effects become negligible and the resistance does not depend on the channel length. In the ballistic limit, the resistance is determined only by the inverse of the number of channels multiplied by the universal quantum resistance $G_{0}^{-1} \equiv h /\left(2 e^{2}\right)=12.9 \mathrm{k} \Omega .{ }^{21}$ The MFP distinguishes the ballistic transport regime from the diffusive one and varies from several nanometers up to micrometer scale, depending on the nature of the various materials and external parameters such as impurities and temperature.

The semiclassical Boltzmann equation method, ${ }^{22}$ which has been used to estimate the MFP, is difficult to apply to the transport problems as the device size becomes small. On the other hand, the quantum transport calculations such as the scattering methods using the plane-wave expansion ${ }^{23-26}$ and real grids, ${ }^{27}$ the embedded-Green's-function (GF) method, ${ }^{28}$ the master-equation method, ${ }^{29}$ and the nonequilibrium GF (NEGF) method ${ }^{30-34}$ suffer from $\sim O\left(N^{3}\right)$ computation and the system size is restricted much less than the MFP. The difficulty to perform quantum transport calculations for large-scale systems prohibits us to study the transport properties from ballistic to diffusive regimes.

We developed a time-dependent wave-packet diffusion (TD-WPD) method ${ }^{35}$ for the quantum transport calculation of huge systems. Based on the linear-response KuboGreenwood formula, we calculate the conductance through time-dependent diffusion coefficients using the timedependent wave-packet diffusion approach ${ }^{36-38}$ combining with molecular-dynamics (MD) simulations. ${ }^{39}$ Using the Chebyshev polynomial expansion for the time-evolution operator for the electron wave-packet motion, we treat the transport properties of huge systems with $O(N)$ high-speed calculations. We can study the electron transport properties of materials from atomistic viewpoints up to 80 million atoms at various temperatures from ballistic to diffusive regimes, including the impurity and electron-phonon scatterings where all the phonon modes are taken into account. 
In this paper, we apply the TD-WPD method to the transport of CNTs. The experiments show that the MFP of CNTs reaches a few hundred nanometers at room temperature. ${ }^{13,14,16}$ Effective-mass theories show that such large MFP is due to the absence of backscattering from impurities $^{40}$ and MFP is determined from the acoustic phonon scatterings. ${ }^{41} \mathrm{We}$ show the transport properties of metallic CNTs depending on the length, temperature, and the Fermi energy from ballistic to diffusive regimes systematically. We also present the transport properties of semiconducting CNTs coupled with metallic electrodes for the channel-length dependence of mobilities and cut-off frequencies at room temperature.

\section{TIME-DEPENDENT WAVE-PACKET DIFFUSION METHOD}

First, we describe the TD-WPD method briefly. The conductivity $\sigma$ at energy $E$ for the Kubo-Greenwood formula, ${ }^{42,43}$

$$
\sigma(E)=\frac{e^{2}}{\Omega} \sum_{n} \delta\left(E-E_{n}\right) \int_{-\infty}^{+\infty}\left\langle n\left|\hat{v}_{z}(t) \hat{v}_{z}(0)\right| n\right\rangle d t,
$$

where $\hat{v}_{z}$ is the electron velocity operator and $\Omega$ is the volume of the system, is expressed using the time-dependent diffusion coefficient,

$$
D\left(E_{n}, t\right)=\frac{\left\langle n\left|[\hat{z}(t)-\hat{z}(0)]^{2}\right| n\right\rangle}{t}
$$

with the velocity correlation ${ }^{42}$

$$
\int_{-\infty}^{+\infty}\left\langle n\left|\hat{v}_{z}(t) \hat{v}_{z}(0)\right| n\right\rangle d t \equiv \lim _{t \rightarrow+\infty} D\left(E_{n}, t\right),
$$

such as the Einstein relation $\sigma=e^{2} \nu(E) \lim _{t \rightarrow+\infty} D(E, t)$. Here the density of states $\nu(E)$ is defined by $\nu(E)$ $=(2 / \Omega) \Sigma_{n} \delta\left(E-E_{n}\right)$.

Taking the maximum value in the time domain as a diffusion coefficient, the resistance $R(L)$ is given by

$$
R(L)=\frac{L}{S} \frac{1}{e^{2} \nu(E) D_{\max }(E, t \rightarrow+\infty)},
$$

where the system length along $z$ axis is $L$ and the crosssectional area is $S$.

In the diffusive regime, since the velocity correlation vanishes after the relaxation time $\tau$ with $\left\langle v(s) v\left(s^{\prime}\right)\right\rangle$ $=\left\langle v^{2}\right\rangle \exp \left(-2\left|s-s^{\prime}\right| / \tau\right)$, we have

$$
D(E)=\left\langle v^{2}\right\rangle \tau=v \ell_{\mathrm{mfp}},
$$

where $\ell_{\mathrm{mfp}}$ represents MFP. Then the resistance is proportional to the system length $L$ and is inversely proportional to $S$, which is the classical Ohm's law. In the ballistic regime, electrons propagate at constant velocity $v$ and $D(E, t)=v^{2} t$ $=v L$. Using $\nu=2 /\left(2 \pi \hbar v / N_{c}\right) S$, where $N_{c}$ is the number of the channels, the conductance $G(L)$ becomes

$$
G(L)=\frac{S}{L} e^{2} \times \frac{2}{\left(2 \pi \hbar v / N_{c}\right) S} \times v L=\frac{2 e^{2}}{h} N_{c},
$$

independent of the length $L$. Thus, if we can evaluate $D(E, t)$ from an atomistic point of views, we can obtain the transport properties in both regimes, in addition to the intermediate quasiballistic regime systematically.

The essence of the TD-WPD method is that, instead of calculating the numerical diagonalization of the matrix $\hat{H}$, the time-dependent diffusion coefficient is evaluated by using wave packets as follows:

$$
D(E, t) \equiv \frac{\left\langle[\hat{z}(t)-\hat{z}(0)]^{2}\right\rangle_{E}}{t},
$$

where we take the statistical average of the operator $\hat{A}=[\hat{z}(t)-\hat{z}(0)]^{2}$ by the formula,

$$
\langle\hat{A}\rangle_{E} \equiv \frac{\sum_{n}\left\langle\Psi_{n}|\delta(E-\hat{H}) \hat{A}| \Psi_{n}\right\rangle}{\sum_{n}\left\langle\Psi_{n}|\delta(E-\hat{H})| \Psi_{n}\right\rangle} .
$$

The propagation length of an electron is defined as $L(E, t) \equiv \sqrt{\left\langle[\hat{z}(t)-\hat{z}(0)]^{2}\right\rangle_{E}}$, where $D(E, t)$ is represented by $D(E, t)=L(E, t)^{2} / t$. Sufficient numbers of initial wave packets are enough for the convergence of $D(E, t)$ to the one that is represented by the complete sets of the eigenfunctions $|n\rangle$. This is suitable for the use of parallel computing. We compute $\left\langle\Psi_{n}|\delta(E-\hat{H}) \hat{A}| \Psi_{n}\right\rangle$ and $\left\langle\Psi_{n}|\delta(E-\hat{H})| \Psi_{n}\right\rangle$ using the Haydock's recursion method. ${ }^{44}$ The trajectories of wave packets $\hat{z}(t)$ are calculated for the Hamiltonian $\hat{H}(t)$ based on the time-evolution operator using the Bessel functions and Chebyshev polynomials of the first kind. ${ }^{36-38}$

The scatterings from phonon vibrations, the main sources of temperature dependence of resistances, are included in the off-diagonal elements of $\hat{H}(t)$ in real-space representation through the time-dependent electron-transfer energies, ${ }^{45}$ $\gamma_{i j}(t)=\gamma_{i j}^{0}\left|\mathbf{R}_{i}^{0}-\mathbf{R}_{j}^{0}\right|^{2} /\left|\mathbf{R}_{i}(t)-\mathbf{R}_{j}(t)\right|^{2}$. We use the MD simulation for the atomic positions $\mathbf{R}_{i}(t)$ at a finite temperature, which enables us to take the realistic various phonon vibration modes into account. Here, we employ the BrennerTersoff potential for the atom-atom interactions, ${ }^{46}$ and solve the coupled equations for the motions of each atom using the velocity Verlet algorithm. ${ }^{39}$ The initial velocities are prepared by the equilibrium of Maxwell's velocity distribution. We take the temperature $T$ to be constant and scale the velocities at each time step. From these MD simulations, the phonondispersion relation is computed as an energy density by taking the two-dimensional Fourier spectra. ${ }^{35,47}$

Figure 1(a) shows the computing times of the time evolution of a wave packet in CNTs as a function of the number of atoms with the time step as $0.1 \times h /(1 \mathrm{eV}) \simeq 0.41$ fs and the summation up to the 12th order of Chebyshev polynomials. We can confirm that the $O(N)$ calculation is realized for the system of up to 80 million atoms. This indicates that we can directly compute the transport properties from an atomic point of view and compare the simulation results with experimental observations of samples which have micron-order 

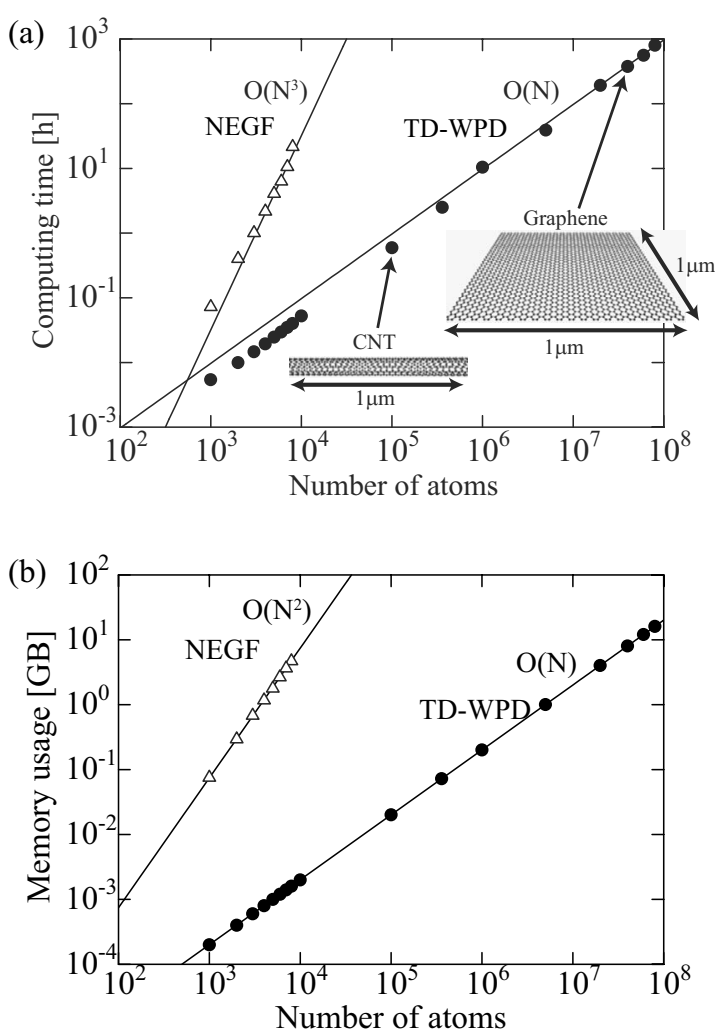

FIG. 1. Logarithm plots of (a) computing times and (b) memory usage to calculate the time evolution of a wave packet in CNTs as a function of number of atoms using the TD-WPD method (black circles) and those by other methods (white triangles). The time step is $0.1 \times h /(1 \mathrm{eV}) \simeq 0.41$ fs and the total evolution time of a wave packet is about $3 \mathrm{ps}$, corresponding to 7200 steps. The number of atoms for CNT with a $1 \mu \mathrm{m}$ length and that for a graphene with a $1 \mu \mathrm{m}^{2}$ area are indicated by arrows, respectively. The two lines in (a) represent the $O\left(N^{3}\right)$ and $O(N)$ dependences while the $O\left(N^{2}\right)$ and $O(N)$ dependences are shown in (b).

lengths. Note that scattering methods or NEGF method ${ }^{30-32}$ needs $O\left(N^{3}\right)$ calculations for the matrix inverse, limiting about 10000 atoms. The memory usage of the TD-WPD method again needs the $O(N)$ due to the storage of the nonzero components of Hamiltonian, compared with the $O\left(N^{2}\right)$ for the NEGF method. Thus the TD-WPD method requires the low resources of computers.

\section{APPLICATIONS TO CARBON NANOTUBE TRANSPORT}

We apply the TD-WPD method to the transport properties of CNTs. In the metallic CNT cases, due to the unique electronic properties with linear dispersion bands at the charge neutrality point originated from the carbon honeycomb lattice structures, the transport properties are characterized by the absence of backscattering from long-range impurities. ${ }^{40}$ Accordingly, CNTs are ideal one-dimensional ballistic conductors and are expected to show high mobility transport properties $^{15,48,49}$ and a long MFP. ${ }^{13,14,50}$

On the other hand, the observed experimental data for the CNTs longer than a few hundred nanometers ${ }^{14,16}$ show dif- fusive transport characters such as the length-dependent resistance. These experimental observations lead us to study the transport properties of CNTs in various transport regimes and determine the MFP quantitatively at various temperatures where the dominant origin of resistance is vibrating phonon scatterings. Up to now, the phonon effects have been studied for specific modes ${ }^{36}$ or using the continuum media model. ${ }^{41,51}$ Here we take various realistic phonon modes thermally excited at a finite temperature to study the realistic phonon-scattering effects on the transport properties of CNTs. We employ the $\pi$-effective Hamiltonian for the phonon scatterings with the time-dependent transfer energies $\gamma(t)$. We take $\gamma^{0}=2.5 \mathrm{eV}$ with an equilibrium carbon-carbon bond length of $0.144 \mathrm{~nm}$. The MD simulations are performed for a $250 \mathrm{~nm}$ (1000 unit cells) armchair CNT subjected to a periodic boundary condition, sufficiently large for the convergence. ${ }^{47,52}$

As for an electron propagation to compute $D(E, t)$ in Eq. (6), we use two initial electron wave packets, localized at one $\pi$ orbital on A and B sublattice around the center part of the CNT. Note that the results do not change when we use the random-phase states as initial wave packets. ${ }^{36}$ Four Maxwell velocity distributions of initial atoms are employed in the MD simulations and thus $2 \times 4$ initial conditions are averaged for $D(E, t)$, where the parallel computing on eight processors are assigned to perform the time-evolution calculation. The summation up to the 12th order of Chebyshev polynomial is sufficient to ensure convergence. The calculations up to $20 \mu \mathrm{m}$ (80 000 unit cells) armchair CNTs are performed with the total evolution time of about $15 \mathrm{ps}$ and the time step of $\Delta t \equiv 0.1 h /(1 \mathrm{eV})=0.41$ fs.

\section{A. Metallic CNT}

\section{Length dependence from ballistic to diffusive regimes}

We study how the transport properties of the metallic CNTs change according to the channel length $L$ from the ballistic to diffusive regimes due to the electron-phonon scatterings. First, we note that the phonon-dispersion relation obtained from the MD simulation ${ }^{35}$ shown in Fig. 2(b) agrees very well with other phonon calculations for CNTs. ${ }^{52}$ We can see three acoustic phonon branches at a low-frequency region, i.e., flexure (F) mode, twist (TW) mode, and longitudinal-acoustic (LA) mode, shown by arrows.

Figure 2(c) shows the calculated length-dependent resistance of the metallic CNTs at $300 \mathrm{~K}$, taking all of the phonon modes for the scatterings. We use $20 \mu \mathrm{m}$ length $(5,5)$ - and $(10,10)$-CNTs and the Fermi energy $E_{F}$ is located at the charge neutrality point. Fixing the channel length to the propagation length $L\left(E_{F}, t\right)$, we plot the resistance $R(L)$ $=L\left(E_{F}, t\right) /\left[e^{2} N\left(E_{F}\right) D\left(E_{F}, t\right)\right]$ as a function of the channel length $L\left(E_{F}, t\right)$, where $N\left(E_{F}\right)\left[=\nu\left(E_{F}\right) S\right]$ is the density of states per unit length of the CNT.

We note that there are three regimes. When the channel length is large enough, the resistance $R(L)$ shows a monotonically increasing behavior for $L$. We can plot a thin solid line to fit to an asymptotic behavior of the calculated result with a linear equation of $R(L)=\rho \cdot L+R_{0}$, where the slope $\rho$ corresponds to the resistivity. We can see that this asymptotic 

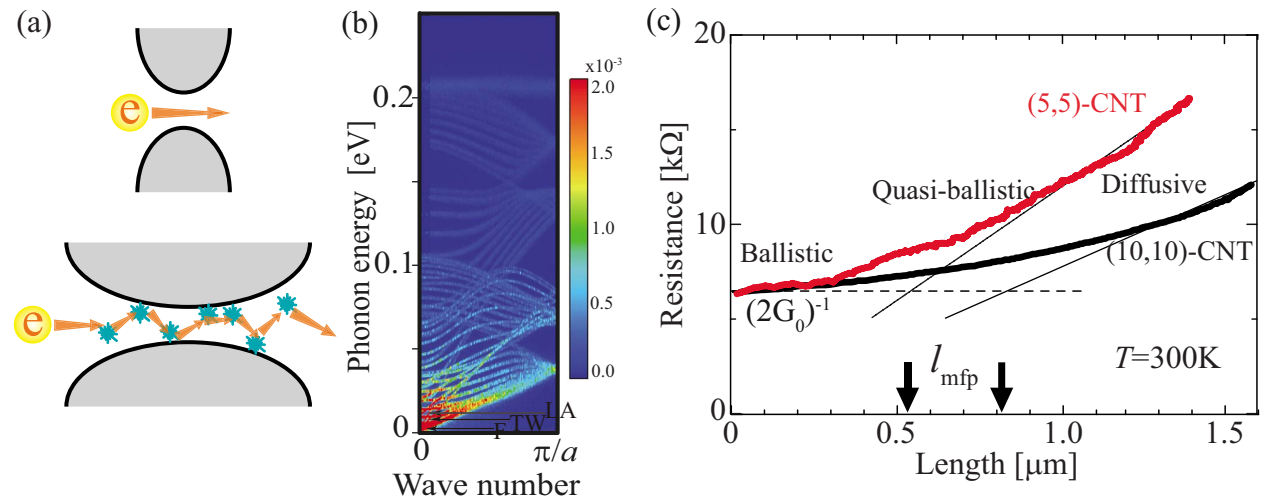

FIG. 2. (Color online) (a) Schematic pictures of ballistic (upper) and diffusive (lower) transport behaviors. (b) Phonon-dispersion relation of the metallic $(10,10)$-CNT. The length of a unit cell is represented by $a$. Flexure (F) mode, twist (TW) mode, and longitudinal-acoustic (LA) mode are shown by arrows. (c) The length dependence of resistance of metallic (5,5)-CNT (red) and (10,10)-CNT (black) from ballistic to diffusive regimes at the charge neutrality point. The thin solid line indicates the diffusive limit, while the thin broken line shows the ballistic limit $\left(2 G_{0}\right)^{-1}=6.45 \mathrm{k} \Omega$, whose intersections represent the MFP $\ell_{\text {mfp }}$ indicated by arrows.

line converges to zero $R_{0}=0$ as the channel length $L$ decreases to zero. This means that this line shows the classical resistance and the asymptotic behavior for large $L$ manifests a typical transport property in the diffusive regime where the system size is much larger than the MFP. Note that this situation, illustrated schematically in the lower picture of Fig. 2(a), is realized when an electron loses the velocity correlation $\langle\hat{v}(t) \hat{v}(0)\rangle$ due to the many scatterings with phonons, resulting in the saturation of the diffusion coefficient $D_{\text {max }}\left(E_{F}\right)$ independent of the time and length.

When the channel length $L$ becomes small to zero, the resistance $R(L)$ becomes independent of the length $L$ and converges to $\left(2 \times 2 e^{2} / h\right)^{-1}=\left(2 G_{0}\right)^{-1}$, where 2 comes from the channel number of the metallic CNTs at the charge neutrality point. This is a typical feature of quantum transport in the ballistic limit and a corresponding asymptotic behavior is shown by the thin broken line.

The thin solid and broken lines intersect at the value $\ell_{\text {mfp }}$ shown in Fig. 2(c) by the thick arrow. We find that this value is very close to the length obtained from $\ell_{\mathrm{mfp}}=D_{\max }\left(E_{F}, t \rightarrow \infty\right) / v_{F}$, which reaches $\ell_{\mathrm{mfp}}=515 \mathrm{~nm}$ for the $(5,5)-\mathrm{CNT}$ at room temperature $(300 \mathrm{~K})$. Here, the Fermi velocity $v_{F}$ is obtained from the slope of the propagation length $L\left(E_{F}, t\right)$ as a function of time $t$ in the vicinity of $t=0$. This shows clearly that the length $\ell_{\mathrm{mfp}}$ obtained from the calculation of $D(E, t)$ corresponds to the MFP, which separates the regimes from ballistic to diffusive.

When we define the regime where the nanotube length is comparable to the MFP $\ell_{\mathrm{mfp}}$, say, $300-800 \mathrm{~nm}$, as a quasiballistic transport regime, the resistance there takes much larger values than those expected from the linear dependence of resistance in the diffusive regime and shows a nonlinear behavior. In other words, we can specify the quasiballistic regime for the regime where the resistance deviates from the diffusive and ballistic asymptotic lines, which is 300-800 $\mathrm{nm}$ for the metallic $(5,5)$-CNTs. For the metallic CNT with a different chirality of $(10,10)$-CNTs, we can see similar behavior of length-dependent resistance from ballistic to diffusive regime as shown by the bold black curve in Fig. 2(c). The quantitative difference between different chiralities is discussed in Sec. III A 3.
These results show that, using the TD-WPD method, we can evaluate the transport properties of CNTs not only in the diffusive and ballistic regimes, but also in the quasiballistic regime from atomistic viewpoints systematically at a finite temperature. It should be noted that, taking the realistic phonon scatterings into account, the resistance is correctly described with the present method in the classical diffusive transport regime without introducing any damping factors for a dominant electron-phonon coupling, which are needed to obtain the relaxation time to estimate the MFP.

\section{Temperature and diameter dependence}

Next, we show the temperature-dependent transport properties of the $(5,5)$-CNTs from the ballistic to diffusive regimes. Figure 3(a) shows the length-dependent resistance for several temperatures ranging from 10 to $300 \mathrm{~K}$. The thin solid asymptotic lines represent the length-dependent behaviors of resistance in the diffusive limit while the asymptotic behaviors in the ballistic regimes are shown by the thin broken lines. We see that the CNT resistances are strongly dependent on the temperature $T$. For example, when we prepare the metallic CNT with $1 \mu \mathrm{m}$ long, then cool it down from 300 to $10 \mathrm{~K}$, the temperature behaviors of transport properties change from diffusive $(300 \mathrm{~K})$ to quasidiffusive $(100 \mathrm{~K})$, up to ballistic $(10 \mathrm{~K})$ regimes. Since the transport experiments of CNTs have frequently been done using the CNT lengths close to $1 \mu \mathrm{m}$, this result suggests that the temperatures are very important parameters for the analyses of the resistance behaviors of CNTs.

In the classical diffusive limit, the continuum model approach based on the effective-mass theory ${ }^{41}$ shows that only TW mode contributes to the backscattering for the armchair CNTs and the MFP $\ell_{\text {mfp }}$ is obtained as follows:

$$
\ell_{\mathrm{mfp}}=\pi \mu\left(\frac{3 a_{\mathrm{cc}} \gamma^{0}}{4 g_{2}}\right)^{2} \frac{d}{k_{B} T},
$$

where $d$ is the diameter of the CNT and $\mu$ and $g_{2}$ are the shear modulus and the deformation potential of CNTs, respectively. When we use the parameters such as $\mu=57.38 \mathrm{~kg} / \mathrm{s}^{2}$ and $g_{2}=0.8-2.7 \mathrm{eV}$ extracted from the data 
(a)

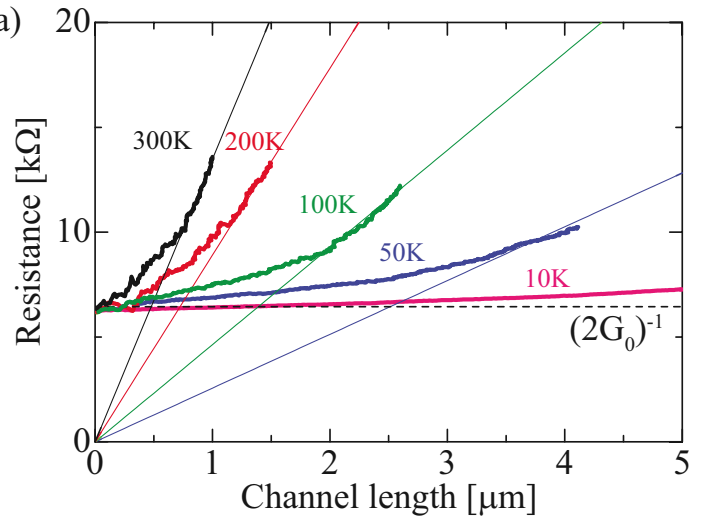

(b)

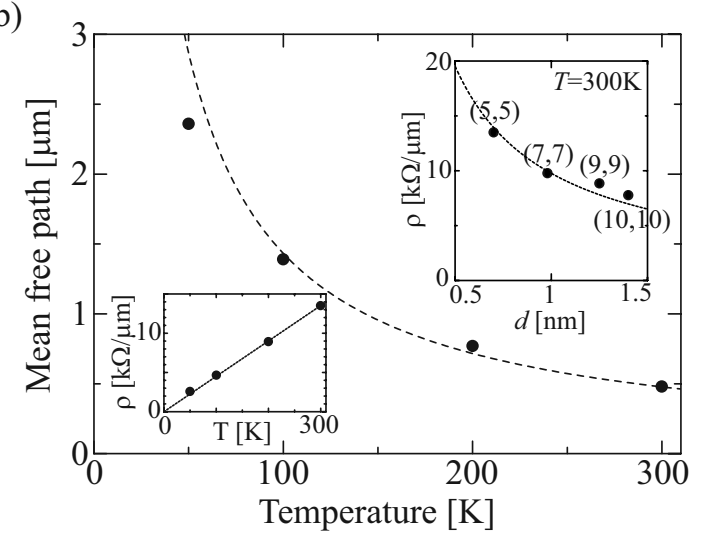

FIG. 3. (Color online) (a) Channel-length dependence of resistance of the $(5,5)$-CNT at several temperatures ranging from 10 to $300 \mathrm{~K}$. Thin solid lines represent the diffusive limit while thin broken line show the ballistic limit. (b) Inelastic MFP of the $(5,5)-\mathrm{CNT}$ due to electron-phonon couplings from 50 to $300 \mathrm{~K}$. The dashed curve represents an inverse temperature dependence, which indicates that the acoustic phonons contribute to the transport properties. (Lower left inset) Temperature dependence of resistivity $\rho$ in the diffusive regime. (Upper right inset) Resistivity $\rho$ of $(5,5)$-, $(7,7)-,(9,9)-$, and $(10,10)-\mathrm{CNTs}$ at $300 \mathrm{~K}$ as a function of diameter d.

of "graphite," since they are not obtained from the CNT experiments nor theoretically, we obtain the MFP larger than a few hundred nanometers even at room temperature and shows the $T^{-1}$ dependence due to scattering by the acoustic phonons. This theory agrees qualitatively with the experiments in the diffusive transport limit. ${ }^{16,53}$

Let us compare the MFPs for several temperatures with those from the effective-mass theory. The obtained MFPs are shown in Fig. 3(b) as a function of temperature. We see an inverse temperature dependence very clearly, which is consistent with the effective-mass theory. In the present TDWPD method, the MFP evaluated at $300 \mathrm{~K}$ reaches about $500 \mathrm{~nm}$. We note that the MFP observed in experiments ranges from $300 \mathrm{~nm}$ to $1.6 \mu \mathrm{m}$ at a low source-drain bias voltage, depending on the chiralities and impurities, etc. $13,14,16,41,51$ From the obtained MFPs, we have the temperature-dependent one-dimensional resistivity $\rho$ defined by $\rho \equiv R(L) / L \approx 1 /\left(2 G_{0} \ell_{\mathrm{mfp}}\right)$, where $2 G_{0}$ corresponds to the two conductive channels of the CNTs at $E_{F}$. In the lower left inset of Fig. 3(b), we show $\rho$ as a function of temperature, ${ }^{54}$ which represents the clear linear dependence, such as $\rho(T)=0.045 T \mathrm{k} \Omega / \mu \mathrm{m}$, in good agreement with other experiments ${ }^{14,16,53}$ and also with the results from effectivemass theories. ${ }^{41}$

The diameter dependence of resistivity is shown in the upper right inset of Fig. 3(b), where we study the resistivity of the (5,5)-, (7,7)-, (9,9)-, and (10,10)-CNTs. We see that the resistivity clearly shows the inversely proportional behavior to the nanotube diameter $d$, consistent with Eq. (8). We find that the data is well fitted by the dashed curve of $\rho=9.765 / d(\mathrm{k} \Omega / \mu \mathrm{m})$, where $d$ is in units of nanometer. From these results, we can deduce the temperature- and diameter-dependent resistivity of metallic CNTs in the diffusive regime due to electron-phonon coupling as follows:

$$
\rho(\mathrm{k} \Omega / \mu \mathrm{m}) \simeq 0.032 \frac{T(\mathrm{~K})}{d(\mathrm{~nm})} .
$$

We note that $\rho$ extracted from experimental results range from 0.024 to $0.121 .^{13,14,16}$ These results show that the present TD-WPD method is effective for the transport properties in various transport regimes at various temperatures. This suggests the possibility to study the temperature dependence of transport properties for other materials.

\section{Energy dependence of mean-free path}

We study the energy dependence of MFP to compare the transport properties in the linear band dispersion region with those in the nonlinear band dispersion region far away from the charge neutrality point. Figure 4(a) shows the calculated MFP of $(5,5)-C N T$ as a function of the energy at several temperatures, where the charge neutrality point is located at $0 \mathrm{eV}$. We see that the MFP becomes very long around $0 \mathrm{eV}$, which increases remarkably as the temperature decreases, i.e., up to about $3 \mu \mathrm{m}$ at $T=50 \mathrm{~K}$. This is due to the suppression of backscattering from the linear band dispersion as shown in the inset of Fig. 4(a).

On the other hand, the MFP drops rapidly down in the higher subband region $(|E| \geq 1.5 \mathrm{eV})$ since new scattering channel bands are open for the conduction of electrons. For example, the MFP with $3 \mu \mathrm{m}$ around $E=0 \mathrm{eV}$ is reduced to $500 \mathrm{~nm}$ around $E=2 \mathrm{eV}$ at $T=50 \mathrm{~K}$ and $500-100 \mathrm{~nm}$ at $T=300 \mathrm{~K}$. Similar energy dependences of the transport behaviors have been obtained in the experiment in CNTs (Ref. 50) and also in the model calculation with static disorders. ${ }^{55}$

Figure 4(b) shows the length dependence of $R(L)$ at $300 \mathrm{~K}$ for $E=0 \mathrm{eV}$ and $E=-2 \mathrm{eV}$, indicated by arrows in Fig. 4(a). When we change the energy from 0 to $-2 \mathrm{eV}$, the resistance decreases to one third, from $\left(2 G_{0}\right)^{-1}$ to $\left(6 G_{0}\right)^{-1}$ in the ballistic limit, which reflects that six effective channels are open at $E=-2 \mathrm{eV}$. As the length of the CNT increases, the behaviors of resistance change significantly. We see that the resistance increases very rapidly, with the slope of resistivity $\rho=31 \mathrm{k} \Omega / \mu \mathrm{m}$ for $E=-2 \mathrm{eV}$, showing that an opening of new scattering channels increase the resistance considerably. On the other hand, an increase in resistance for $E=0 \mathrm{eV}$ is much slow with $\rho=13 \mathrm{k} \Omega / \mu \mathrm{m}$. So these two resistances cross with the length close to $200 \mathrm{~nm}$. We also 

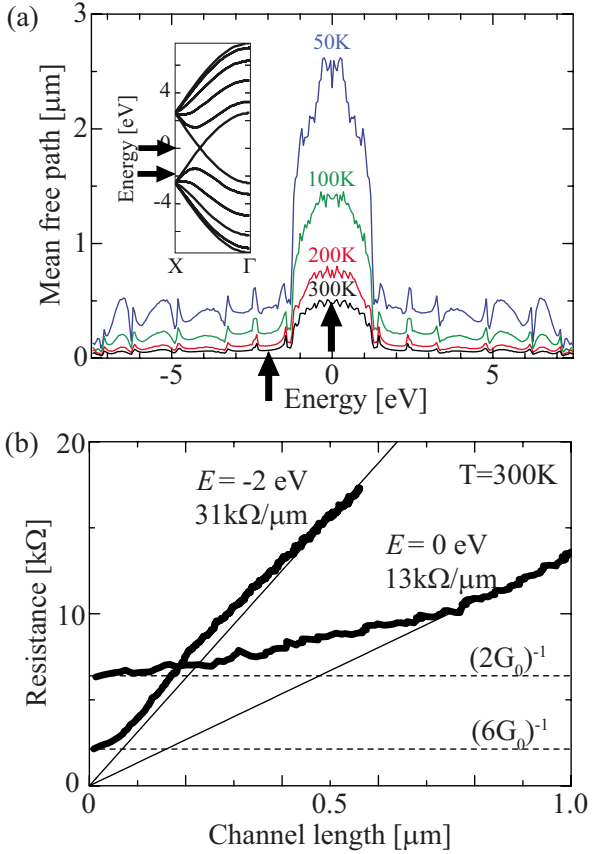

FIG. 4. (Color online) (a) Mean-free path $\ell_{\mathrm{mfp}}$ as a function of energy $E$ for the $(5,5)$-CNT at temperatures from $T=50$ to $300 \mathrm{~K}$. (Left inset) Band structure of the $(5,5)-\mathrm{CNT}$. Two arrows indicate $E=0$ (charge neutrality point) and $E=-2 \mathrm{eV}$ where the new bands appear. (b) The resistance vs channel length at $E=0$ and $-2 \mathrm{eV}$ corresponding to the arrows in (a). The thin solid lines represent the asymptotic behaviors in the diffusive regimes for $E=0$ and $-2 \mathrm{eV}$ while the resistances in the ballistic limit are shown by broken lines and are equal to $\left(2 G_{0}\right)^{-1}$ for $E=0$ and $\left(6 G_{0}\right)^{-1}$ for $E=-2 \mathrm{eV}$ corresponding to the number of conductive channels.

note that the MFP for $E=-2 \mathrm{eV}$ is almost vanishing and thus the ballistic transport regime is difficult to observe in experiments for any nanotube length when we set the energy corresponding to $E=-2 \mathrm{eV}$.

\section{B. Semiconducting CNT}

\section{Length dependence of mobility and cut-off frequency}

Here, we consider the transport properties of the semiconducting CNT, which is applicable to the field-effect-transistor (FET) devices. The mobility and cut-off frequency of $(10,0)$ CNT FETs are investigated from ballistic to diffusive regimes at room temperature using the TD-WPD method.

In the semiconducting CNT, the contacts with metallic electrodes are very important, since Schottky barriers are formed due to the charge redistribution close to the contacts. Here we treat this effect using a phenomenological planar gate model as shown in the inset of Fig. 5(a). The gate voltage effects on the potential energy, included in $\hat{H}(t)$ as an on-site energy for each atom, are obtained as follows.

We consider that the CNT with the radius $R$ is put along the $z$ axis and the metallic gold electrode is attached at the right side, which is surrounded by a dielectric material with $\varepsilon$ uniformly. The distance between the CNT and the gate substrate is $R_{s}$. The potential of semiconducting CNTs attached (a)
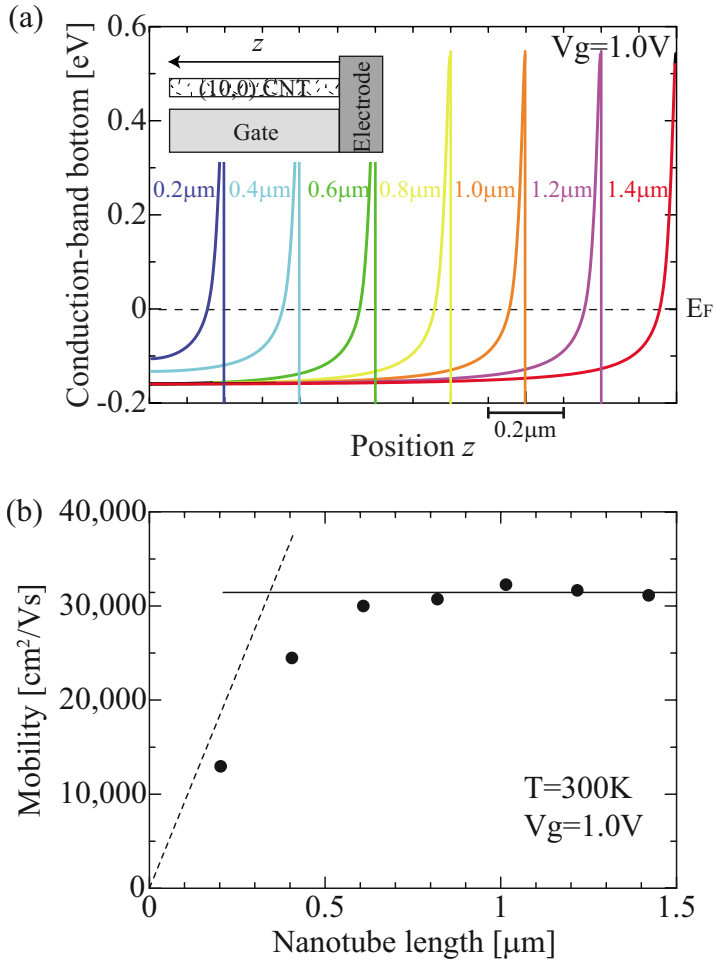

(c)

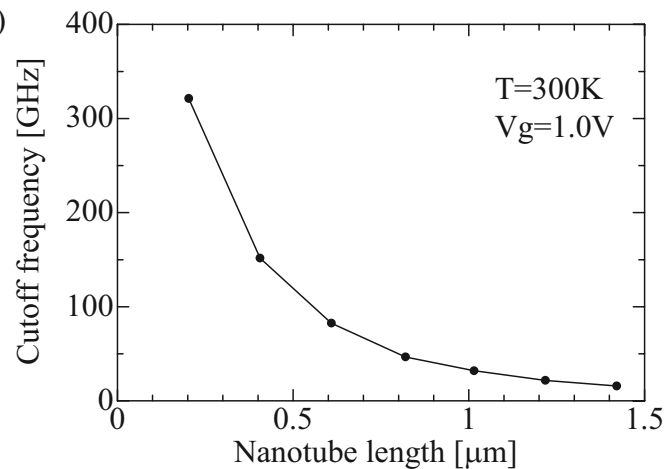

FIG. 5. (Color online) (a) Conduction-band bottom of the semiconducting $(10,0)$-CNT at $V_{g}=1.0 \mathrm{~V}$ as a function of position along the CNTs with channel lengths from $L=0.2$ to $1.4 \mu \mathrm{m}$. The Schottky barrier is formed at the right-side contact. Fermi energy is represented by the broken line. (Inset) Schematic picture of the CNT-FET configuration. (b) Channel-length dependence of pseudomobility of the CNT-FET at $300 \mathrm{~K}$. A gate voltage of $1.0 \mathrm{~V}$ is applied to the CNT. Thin solid line represents the diffusive limit while thin broken line shows the ballistic limit. (c) Cutoff frequency as a function of the CNT length.

to the metallic electrodes is obtained from the self-consistent calculation of the Poisson equation, ${ }^{56}$ which relates the total potential $\phi(z)$ to the charge density $\rho(z)$ of the CNT under an applied gate voltage $V_{g}$,

$$
\phi_{q}=U_{q} \rho_{q}+M_{q} \Psi_{q} .
$$

Here $\phi_{q}, \rho_{q}$, and $\Psi_{q}$ are the Fourier transformation of $\phi(z), \rho(z)$, and the gate bias $\Psi(z) \equiv V_{g}$, respectively. Using the modified Bessel functions $I_{0}$ and $K_{0}$, the coefficients $U_{q}$ and $M_{q}$ are given by the analytic forms of $U_{q}=2\left\{I_{0}(q R) K_{0}(q R)-K_{0}\left(2 q R_{s}\right)\right\} / \varepsilon$ and $M_{q}=\exp \left(-|q| R_{s}\right)$. 
The charge neutrality level $E_{0}(z)$ of the CNT measured from $E_{F}=0 \mathrm{eV}$ is related to the total potential $\phi(z)$ such as

$$
E_{0}(z)+e \phi(z)=\Delta W,
$$

where $\Delta W$ is one of the fitting parameter independent of $z$. Since the charge neutrality level is given, the $z$-dependent charge density can be obtained from

$$
\rho(z)=\rho_{0}(z)+e \frac{8 \pi R}{3 \sqrt{3} a_{\mathrm{cc}}^{2}}\left[D_{0} E_{0}(z) \exp \left(-\frac{z}{d}\right)\right] \operatorname{sgn}(z)
$$

and

$$
\rho_{0}(z)=e \int N(E) \operatorname{sgn}(E) f\left[\left\{E-E_{0}(z)\right\} \operatorname{sgn}(E)\right] d E .
$$

The pinning strength is represented by $D_{0}$ and we take an effective decay constant $d$ of the surface states to be about 2 $\mathrm{nm} .{ }^{57} N(E)[=\nu(E) S]$ represents the density of states per unit length and $f(E)$ is the Fermi distribution function. Equations (10)-(12) are solved self-consistently to obtain $\phi(z)$. In order to realize the boundary condition at $\phi(z=0)=0$, we employ an antisymmetric condition, $\Psi(z)=V_{g} \operatorname{sgn}(z)$ and $E_{0}(z)+e \phi(z)=\Delta W \operatorname{sgn}(z)$, instead of $\Psi(z)=V_{g}$ and Eq. (11). We assume that the $(10,0)$-CNT is surrounded by a dielectric material of $\varepsilon=5.0$ and the distance is set as $R_{s}=19 \mathrm{~nm}$. To fit the experimental values, we adopt the parameters $D_{0}=0.1,{ }^{56}$ and $\Delta W=0.65 \times E_{g} / 2$, where the energy band gap $E_{g}$ of the $(10,0)$-CNT is equal to $0.8 \mathrm{eV}$.

Figure 5(a) shows the effective potential in the vicinity of contact, thus conduction-band bottom as a function of position $z$ along the CNTs with various channel lengths ranging from 0.2 to $1.4 \mu \mathrm{m}$ for a gate voltage $V_{g}=1.0 \mathrm{~V}$. We can see that the Schottky barrier is formed at the contact to electrode, whose effects range up to $100 \mathrm{~nm}$ inside the CNTs. This is due to the weak screening of CNTs as a lowdimensional material. These potential energies are included in the diagonal elements of $\hat{H}(t)$ for each atomic position.

Let us see how the mobility changes according to the length of the CNT from the ballistic to the diffusive regimes. We define the pseudomobility $\mu^{*}$ by the following equations within the effective-mass approximation:

$$
\mu^{*} \equiv \frac{e \tau}{m^{*}} \quad \text { with } \quad \tau=\frac{D_{\max }\left(V_{g}\right)}{v^{2}},
$$

where $\tau$ represents the scattering time and the velocity $v$ is obtained from $0.59 \mathrm{~nm} / \mathrm{fs}$ for $V_{g}=1.0 \mathrm{~V}$. We approximate the bottom of the conduction band by a parabolic curve and the effective mass of electrons, which is defined by $1 / m^{*} \equiv d^{2} E(k) / \hbar^{2} d k^{2}$ is evaluated as $m^{*} \simeq 0.296 \times 10^{-31} \mathrm{~kg}$.

Note that the calculated $\mu^{*}$ is the pseudomobility, since the mobility is generally defined only in the diffusive regime, not in the (quasi-)ballistic regime. The important point here is that there are two factors to determine the time $\tau$. One is the relaxation time we expect in the diffusion regime due to the scatterings with phonons. The other is the time at which electrons reach the contact where the potential barrier is formed. Therefore, if the channel length $L$ is short enough, we expect that the pseudomobility $\mu^{*}$ will be proportional to $L$ and approaches zero for the limiting $L=0$ case. We may call such a regime as a (quasi-)ballistic regime since the electron mobility is determined by the device structure itself. In the present calculation, we put two initial localized wave packets at the left edge of CNT and then $D_{\max }$ and $\mu^{*}$ are obtained from the time-dependent calculations of $D(E, t)$. The MD simulations are performed for 470 unit cells zigzag $(10,0)$-CNT subjected to a periodic boundary condition at $300 \mathrm{~K}$. Two Maxwell distributions of initial atom velocities are employed in the simulation for the phonons.

Figure 5(b) shows the calculated pseudomobility $\mu^{*}$ vs nanotube length $L$ for the $(10,0)$-CNT FET devices applying a gate voltage of $V_{g}=1.0 \mathrm{~V}$ at $300 \mathrm{~K}$. The transport properties are studied as a function of nanotube lengths ranging from $200 \mathrm{~nm}$ up to $1.4 \mu \mathrm{m}$. We see that the obtained $\mu^{*}$ changes the characteristics gradually from $L \approx 600 \mathrm{~nm}$. Since the pseudomobility takes the value of $\mu^{*} \approx 30000 \mathrm{~cm}^{2} / \mathrm{V} \mathrm{s}$ independent of the length, as shown by the straight line in Fig. 5(b), we can say that the diffusive regime is realized where the nanotube length is longer than $600 \mathrm{~nm}$ and the mobility is determined by the electronphonon scatterings there. This corresponds to the intrinsic mobility of the CNT, which is observable in experiments, not to be related to the device structure such as contacts to electrodes. We note that the reported experimental values of mobility range from 1000 to $79000 \mathrm{~cm}^{2} / \mathrm{V} \mathrm{s}$, depending on the various samples. ${ }^{15,48,49,58,59}$ The present results are also consistent with the previous theoretical studies. ${ }^{48}$

On the other hand, when the nanotube length becomes smaller than $L=600 \mathrm{~nm}$, the pseudomobility decreases as the nanotube length decreases. This clearly shows that the electron motions are significantly affected by the Schottky barrier formation due to the contact effects from the geometrical device structure, whose effects range up to $100 \mathrm{~nm}$ or more. Therefore we can say that the system is in the quasidiffusive and the ballistic regimes.

The MFP obtained from

$$
\ell_{\mathrm{mfp}}\left(V_{g}\right)=\frac{D_{\text {max }}\left(V_{g}, t \rightarrow \infty\right)}{v}
$$

including the electron-phonon coupling and the Schottky barrier from the contact geometry is evaluated as $343 \mathrm{~nm}$ for the semiconducting $(10,0)$-CNT. This also corresponds to the intersection value where the two asymptotic lines for the diffusive and ballistic limit cross in Fig. 5(b), where the broken line in the ballistic regime is plotted as a linear length dependence that the pseudomobility $\mu^{*}$ shows, such as $\mu=e L /\left(m^{*} v\right)$. We note that this MFP is shorter than that of metallic (5,5)-CNTs (Ref. 35) even though these CNTs have almost the same radius $R$. This is because opening band gap disrupts the linear band dispersion around the Fermi energy in the semiconducting CNT case.

These observations show that the transport properties of semiconducting $(10,0)$-CNT change with the channel length about $L=600 \mathrm{~nm}$ from the diffusive to quasiballistic regime, and about $L=350 \mathrm{~nm}$ from quasiballistic to ballistic regimes, though these values are strongly affected by the effect of contact to electrodes. Since the observed mobility would change dramatically, careful treatment is needed for the 
analyses of the transport properties in the different transport regimes.

Finally, we estimate the cut-off frequency, which is a standard criterion for the high-speed performance of transistors. High cut-off frequency is essential to realize the high-speed response of devices. Recent experimental works have demonstrated the cut-off frequencies up to $50 \mathrm{GHz}$ using the CNT-FETs with a few hundred nanometers channel length, ${ }^{60-64}$ and the phenomenological law has been also obtained. ${ }^{65}$ Although the cut-off frequency of CNT-FET with a $2 \mathrm{~nm}$ gate is estimated to reach as high as $1.6 \mathrm{THz}$ using the nonequilibrium Green's-function method, ${ }^{66}$ the length dependence of the cut-off frequency from the ballistic to diffusive regimes has not been clarified yet theoretically. Therefore, here we investigate the cut-off frequency using the TDWPD method. The cut-off frequency $f_{T}$ is related to the time $\tau_{T}$ in which electron propagates from the source to the drain electrodes,

$$
f_{T} \equiv \frac{1}{2 \pi \tau_{T}} .
$$

Here, $\tau_{T}$ is obtained using the diffusion coefficient in a low source-drain bias limit, ${ }^{67}$

$$
\tau_{T}=\frac{L^{2}}{D_{\max }\left(E_{F}\right)},
$$

where the channel length is denoted by $L$.

We show the cut-off frequencies vs channel length $L$ for the (10,0)-CNT FET device at room temperature in Fig. 5(c). The applied gate voltage is set to $1.0 \mathrm{~V}$. We see that the cut-off frequency increases with the device size decreasing. The simulated result reveals that the frequency achieves 300 $\mathrm{GHz}$ using the $0.2-\mu \mathrm{m}$-long CNTs. We note that the frequency increases with the diameter of the CNT increases. We can estimate that when $v=0.59 \mathrm{~nm} / \mathrm{fs}, f_{T}$ will reach at $f_{T}=1 \mathrm{THz}$ for $L=100 \mathrm{~nm}$. The present simulation gives very useful information to design the CNT devices with high mobility and with high cut-off frequency.

\section{SUMMARY}

In the present paper, we developed an order- $N[O(N)]$ calculation method for the quantum electron transport of huge systems up to 80 million atoms. This method provides us the conductance and resistance through the timedependent diffusion coefficients based on the KuboGreenwood formula. Combining the motions of timedependent wave packets together with a molecular-dynamics simulation, we derive the TD-WPD approach to calculate conductance. With this TD-WPD method, we can study the temperature-dependent electron transport properties of materials from atomistic viewpoints from ballistic to diffusive regimes, including both the effects of impurity scatterings and electron-phonon couplings.

We applied the present method for the transport properties of metallic and semiconducting CNTs with various lengths at various temperatures. In the metallic CNTs, we evaluated the length dependence of transport properties from ballistic to diffusive regimes at various temperatures. The obtained temperature-dependent mean-free path and resistance are in good agreements with recent experiments. The mean-free path reaches about $500 \mathrm{~nm}$ and increases up to several micrometers at low temperature. Furthermore, we found that, in the quasiballistic regime which exists with lengths between 300 and $800 \mathrm{~nm}$ at room temperatures, the resistances take larger values than expected. In the semiconducting CNTs, the mobilities are affected strongly by contacts with metallic electrodes since the Schottky barriers are formed, whose effects range up to $100 \mathrm{~nm}$ inside the CNTs. We found that the obtained mobilities are maximally about $30000 \mathrm{~cm}^{2} / \mathrm{V} \mathrm{s}$ at room temperature and cut-off frequencies of $300 \mathrm{GHz}$ form semiconducting $(10,0)-C N T s$. These results provide useful information for the design of CNT-FET devices.

\section{ACKNOWLEDGMENTS}

We acknowledge useful suggestions from T. Nakanishi, S. Roche, T. Ikeshoji, and F. Nihey. This work was supported by the Next Generation Super Computing Project, Nanoscience Program, and a Grant-in-Aid for Scientific Research from the Ministry of Education, Culture, Sports, Science and Technology of Japan. The computation in this work has been done in part using the facilities of the Supercomputer Center, Institute for Solid State Physics, University of Tokyo, and the "Interdisciplinary Computational Science Program" at the Center for Computational Sciences, University of Tsukuba.
*Present address: Department of Physics, University of Tokyo, 7-3-1 Hongo, Bunkyo-ku, Tokyo 113-0033, Japan; ishii@bk.tsukuba.ac.jp

${ }^{1}$ H. Ohnishi, Y. Kondo, and K. Takayanagi, Nature (London) 395, 780 (1998).

${ }^{2}$ N. Agraï, C. Untiedt, G. Rubio-Bollinger, and S. Vieira, Phys. Rev. Lett. 88, 216803 (2002).

${ }^{3}$ Y. Okawa and M. Aono, Nature (London) 409, 683 (2001).

${ }^{4}$ Y. Cui and C. M. Lieber, Science 291, 851 (2001).

${ }^{5}$ Y. Wu, J. Xiang, C. Yang, W. Lu, and C. M. Lieber, Nature (London) 430, 61 (2004).
${ }^{6}$ O. Kubo, Y. Shingaya, M. Nakaya, M. Aono, and T. Nakayama, Appl. Phys. Lett. 88, 254101 (2006).

${ }^{7}$ S. J. Tans, A. R. M. Verschueren, and C. Dekker, Nature (London) 393, 49 (1998).

${ }^{8}$ R. Martel, T. Schmidt, H. R. Rhea, T. Herte, and Ph. Avouris, Appl. Phys. Lett. 73, 2447 (1998).

${ }^{9}$ C. Zhou, J. Kong, and H. Dai, Appl. Phys. Lett. 76, 1597 (2000).

${ }^{10}$ A. Bachtold, P. Hadle, T. Nakanishi, and C. Dekker, Science 294, 1317 (2001)

${ }^{11}$ V. Derycke, R. Martel, J. Appenzeller, and Ph. Avouris, Nano Lett. 1, 453 (2001). 
${ }^{12}$ F. Nihey, H. Hongo, Y. Ochiai, M. Yudasaka, and S. Iijima, Jpn. J. Appl. Phys., Part 2 42, L1288 (2003).

${ }^{13}$ A. Javey, J. Guo, M. Paulsson, Q. Wang, D. Mann, M. Lundstrom, and H. Dai, Phys. Rev. Lett. 92, 106804 (2004).

${ }^{14}$ J.-Y. Park, S. Rosenblatt, Y. Yaish, V. Sazonova, H. Üstünel, S. Braig, T. A. Arias, P. W. Brouwer, and P. L. McEuen, Nano Lett. 4, 517 (2004).

${ }^{15}$ T. Dürkop, S. A. Getty, E. Cobas, and M. S. Fuhrer, Nano Lett. 4, 35 (2004).

${ }^{16}$ M. S. Purewal, B. H. Hong, A. Ravi, B. Chandra, J. Hone, and P. Kim, Phys. Rev. Lett. 98, 186808 (2007).

${ }^{17}$ K. S. Novoselov, A. K. Geim, S. V. Morozov, D. Jiang, Y. Zhang, S. V. Dubonos, I. V. Grigorieva, and A. A. Firsov, Science 306, 666 (2004).

${ }^{18}$ F. Miao, S. Wijeratne, Y. Zhang, U. C. Coskun, W. Bao, and C. N. Lau, Science 317, 1530 (2007).

${ }^{19}$ M. Y. Han, B. Özyilmaz, Y. Zhang, and P. Kim, Phys. Rev. Lett. 98, 206805 (2007).

${ }^{20}$ K. I. Bolotin, K. J. Sikes, Z. Jiang, M. Klima, G. Fudenberg, J. Hone, P. Kim, and H. L. Stormer, Solid State Commun. 146, 351 (2008)

${ }^{21}$ B. J. van Wees, H. van Houten, C. W. J. Beenakker, J. G. Williamson, L. P. Kouwenhoven, D. van der Marel, and C. T. Foxon, Phys. Rev. Lett. 60, 848 (1988).

${ }^{22}$ D. K. Ferry, Semiconductor Transport (Taylor \& Francis, London, 2000).

${ }^{23}$ K. Hirose and M. Tsukada, Phys. Rev. Lett. 73, 150 (1994); Phys. Rev. B 51, 5278 (1995); N. Kobayashi, M. Brandbyge, and M. Tsukada, ibid. 62, 8430 (2000); N. Kobayashi, M. Aono, and M. Tsukada, ibid. 64, 121402 (2001); K. Hirose, N. Kobayashi, and M. Tsukada, ibid. 69, 245412 (2004).

${ }^{24}$ N. D. Lang, Phys. Rev. B 52, 5335 (1995).

${ }^{25}$ M. Di Ventra, S. T. Pantelides, and N. D. Lang, Phys. Rev. Lett. 84, 979 (2000).

${ }^{26}$ H. Joon Choi and J. Ihm, Phys. Rev. B 59, 2267 (1999).

${ }^{27}$ Y. Fujimoto and K. Hirose, Phys. Rev. B 67, 195315 (2003).

${ }^{28}$ D. Wortmann, H. Ishida, and S. Blügel, Phys. Rev. B 66, 075113 (2002).

${ }^{29}$ R. Gebauer and R. Car, Phys. Rev. Lett. 93, 160404 (2004); Phys. Rev. B 70, 125324 (2004).

${ }^{30}$ S. Datta, Electronic Transport in Mesoscopic Systems (Cambridge University Press, Cambridge, 1995).

${ }^{31}$ H. Haug and A. P. Jauho, Quantum Kinetics in Transport and Optics of Semiconductors, Springer Series in Solid State Sciences Vol. 123 (Springer, New York, 1996).

${ }^{32}$ J. Taylor, H. Guo, and J. Wang, Phys. Rev. B 63, 245407 (2001).

${ }^{33}$ M. Brandbyge, J.-L. Mozos, P. Ordejon, J. Taylor, and K. Stokbro, Phys. Rev. B 65, 165401 (2002).

${ }^{34}$ Y. Xue, S. Datta, and M. A. Ratner, Chem. Phys. 281, 151 (2002).

${ }^{35}$ H. Ishii, N. Kobayashi, and K. Hirose, Appl. Phys. Express 1, 123002 (2008); J. Vac. Sci. Technol. B 27, 882 (2009).

${ }^{36}$ S. Roche, J. Jiang, F. Triozon, and R. Saito, Phys. Rev. Lett. 95, 076803 (2005).

${ }^{37}$ T. Markussen, R. Rurali, M. Brandbyge, and A.-P. Jauho, Phys. Rev. B 74, 245313 (2006).

${ }^{38}$ H. Ishii, N. Kobayashi, and K. Hirose, Phys. Rev. B 76, 205432
(2007).

${ }^{39}$ W. C. Swope, H. C. Andersen, P. H. Berens, and K. R. Wilson, J. Chem. Phys. 76, 637 (1982).

${ }^{40}$ T. Ando and T. Nakanishi, J. Phys. Soc. Jpn. 67, 1704 (1998).

${ }^{41}$ H. Suzuura and T. Ando, Phys. Rev. B 65, 235412 (2002).

${ }^{42}$ R. Kubo, J. Phys. Soc. Jpn. 12, 570 (1957).

${ }^{43}$ D. A. Greenwood, Proc. Phys. Soc. London 71, 585 (1958).

${ }^{44}$ R. Haydock, V. Heine, and M. J. Kelly, J. Phys. C 5, 2845 (1972).

${ }^{45}$ W. A. Harrison, Electronic Structure and the Properties of Solids: The Physics of the Chemical Bond (Dover, New York, 1989).

${ }^{46}$ D. W. Brenner, Phys. Rev. B 42, 9458 (1990).

${ }^{47}$ J. Shiomi and S. Maruyama, Phys. Rev. B 73, 205420 (2006).

${ }^{48}$ V. Perebeinos, J. Tersoff, and P. Avouris, Phys. Rev. Lett. 94, 086802 (2005)

${ }^{49}$ S. Rosenblatt, Y. Yaish, J. Park, J. Gore, V. Sazonova, and P. L. McEuen, Nano Lett. 2, 869 (2002).

${ }^{50}$ B. Stojetz, C. Miko, L. Forró, and C. Strunk, Phys. Rev. Lett. 94, 186802 (2005).

${ }^{51}$ R. A. Jishi, M. S. Dresselhaus, and G. Dresselhaus, Phys. Rev. B 48, 11385 (1993).

${ }^{52}$ R. Saito, G. Dresselhaus, and M. S. Dresselhaus, Physical Properties of Carbon Nanotubes (Imperial College Press, London, 1998).

${ }^{53}$ C. L. Kane, E. J. Mele, R. S. Lee, J. E. Fischer, P. Petit, H. Dai, A. Thess, R. E. Smalley, A. R. M. Verschueren, S. J. Tans, and C. Dekker, Europhys. Lett. 41, 683 (1998).

${ }^{54}$ H. Ishii, F. Triozon, N. Kobayashi, K. Hirose, and S. Roche, C. R. Phys. 10, 283 (2009).

${ }^{55}$ F. Triozon, S. Roche, A. Rubio, and D. Mayou, Phys. Rev. B 69, 121410(R) (2004).

${ }^{56}$ T. Nakanishi, A. Bachtold, and C. Dekker, Phys. Rev. B 66, 073307 (2002).

${ }^{57}$ F. Léonard and J. Tersoff, Phys. Rev. Lett. 84, 4693 (2000).

${ }^{58} \mathrm{G}$. Pennington and N. Goldsman, Phys. Rev. B 68, 045426 (2003).

${ }^{59}$ X. Zhou, J.-Y. Park, S. Huang, J. Liu, and P. L. McEuen, Phys. Rev. Lett. 95, 146805 (2005).

${ }^{60}$ S. Li, Z. Yu, S.-F. Yen, W. C. Tang, and P. J. Burke, Nano Lett. 4, 753 (2004).

${ }^{61}$ Y.-M. Lin, K. A. Jenkins, A. Valdes-Garcia, J. P. Small, D. B. Farmer, and P. Avouris, Nano Lett. 9, 422 (2009).

${ }^{62}$ C. Kocabas, S. Dunham, Q. Cao, K. Cimino, X. Ho, H.-S. Kim, D. Dawson, J. Payne, M. Stuenkel, H. Zhang, T. Banks, M. Feng, S. V. Rotkin, and J. A. Rogers, Nano Lett. 9, 1937 (2009).

${ }^{63}$ J. Chaste, L. Lechner, P. Morfin, G. Féve, T. Kontos, J.-M. Berroir, D. C. Glattli, H. Happy, P. Hakonen, and B. Placais, Nano Lett. 8, 525 (2008).

${ }^{64}$ A. Le Louarn, F. Kapche, J.-M. Bethoux, H. Happy, G. Dambrine, V. Derycke, P. Chenevier, N. Izard, M. F. Goffman, and J.-P. Bourgoin, Appl. Phys. Lett. 90, 233108 (2007).

${ }^{65}$ P. J. Burke, Solid-State Electron. 48, 1981 (2004).

${ }^{66}$ K. Alam and R. Lake, Appl. Phys. Lett. 87, 073104 (2005).

${ }^{67}$ S. M. Sze, Semiconductor Devices: Physics and Technology, 2nd ed. (Wiley, Hoboken, New Jersey, 2001). 